\title{
Humor im radiologischen Unterricht
}

“First Laugh, Then Think": Appetizers for Medical Student Education in Radiology

\section{Dewey}

\section{Institut}

Radiologie, Charité - Universitätsmedizin Berlin, Humboldt Universität und Freie Universität zu Berlin

Bibliografie

DOI http://dx.doi.org/10.1055/

s-0033-1335852

Fortschr Röntgenstr 2013; 185: 812-815 @ Georg Thieme Verlag KG Stuttgart · New York · ISSN 1438-9029

\section{Korrespondenzadresse}

Prof. Marc Dewey

Klinik für Radiologie, Charité Universitätsmedizin Berlin, Humboldt Universität und Freie Universität zu Berlin

Charitéplatz 1

10117 Berlin

Tel.: ++49/30/450527296

Fax: $++49 / 30 / 450527996$

dewey@charite.de
Was ist gute Lehre? Diese Frage stellt sich immer wieder bei der praktischen Planung eines neuen Seminars, Praktikums oder einer neuen Vorlesung. Gute Lehre sollte ein neues Maß von Verstehen bei den Studenten erzielen und gerade nicht alle letzten Details versuchen zu vermitteln. Wie könnte dies erreicht werden? Ich möchte im Folgenden einen pragmatischen Ansatz zum Erzeugen von „Verstehen“ vorstellen, der geeignet sein könnte, radiologische Lehrveranstaltungen zu bereichern [1].

Der jährlich für unkonventionelle Forschung verliehene Ig-Nobelpreis soll erst zum Lachen bringen und dann nachdenklich machen ("First make people laugh, and then make them think") [2 -5]. Lässt sich dieser Ansatz möglicherweise auch nutzen, um in der akademischen Lehre Medizinstudenten stärker für die Radiologie zu interessieren? Vor besonderen Herausforderungen stehen Lehrende bei großen Studentengruppen, die so gut wie keine Möglichkeit zur aktiven Teilnahme bieten [6]. Könnte man hier mit überraschendem Humor die Zuhörer zum Lachen bringen, um so ihr Interesse für die Radiologie zu wecken, sie zu motivieren und sie zum eigenständigen Weiterlernen anzuregen [7]?

Seit 3 Jahren verwende ich in Vorlesungen und Seminaren Röntgenaufnahmen, Ultraschallbilder, Computertomografien (CT) sowie Magnetresonanztomografien (MRT) von Lebensmitteln. $\mathrm{Zu}$ den abgebildeten Gegenständen gehören Obst wie beispielsweise unterschiedlich reife Bananen ( $\bullet$ Abb. 1 ) oder Äpfel mit und ohne Druckstellen ( $\bullet$ Abb.2) sowie rohe und hart bzw. weich gekochte Eier ( 0 Abb. 3). Diese Bilder werden den Studenten zum Beispiel während der Einführungsveranstaltungen zur Radiologie oder Akutdiagnostikvorlesung mit Fragen wie „Welche Banane oder welchen Apfel ... würden Sie gerne essen wollen?“ oder „Wie hätten unsere Großmütter ein hart gekochtes von einem weich gekochten Ei unterschieden?" Solche Bilder dienen zunächst der unterhaltsamen Einstimmung und gleichzeitig werden nachfolgend die weitere Bedeutung bestimmter Aspekte der Radiologie aufgezeigt [8]. Anhand der Bilder sollen die Studenten lernen, welche Eigenschaften ein Gewebe aufweisen muss, damit es auf radiologischen Bildern in einer bestimmten Weise abgebildet wird ( $\bullet$ Abb. 1-3).

Diese Herangehensweise könnte auch zu der von Nyce et al. geforderten expliziten Vermittlung der Grundannahmen und routinemäßig ablaufenden mentalen Prozesse von Experten im Studentenunterricht beitragen, die häufig nicht berücksichtigt werden, da man die Kenntnis hierüber einfach voraussetzt. Die Lehre bietet uns zudem eine gute Gelegenheit, bei Medizinstudenten mit falschen Vorstellungen über unser Fachgebiet aufzuräumen [9]. Dies ist wichtig, damit alle zukünftigen Ärzte sich der Bedeutung der Radiologie als „Basisfach der Medizin“ [10] und des Beitrags, den Radiologen leisten, bewusst sind. Wer als Lehrer mit Leidenschaft bei der Sache ist und seine Studenten begeistert [11], kann durch humorvolle Materialien verstärkt Interesse wecken [7] und auf diese Weise Denkprozesse und eine interaktive Diskussion anstoßen und so auch mit der verbreiteten Vorstellung aufräumen, dass die Radiologie eine öde Angelegenheit sei, die nur in dunklen Räumen stattfindet [9]. Mit den hier vorgestellten Bildern unterschiedlicher radiologischer Modalitäten können wir Medizinstudenten auch die Angst vor den vermeintlich hohen Anforderungen an Kenntnisse in der Physik nehmen, welche Studenten eventuell als eine unüberwindliche Hürde ansehen und davon abhält, sich für die Radiologie zu entscheiden [9]. Außerdem müssen im Rahmen der Ausbildung angehender Ärzte die Prinzipien der Magnetresonanzverfahren und deren klinische Anwendung durch das Querschnittsfach Radiologie vermittelt werden [12]. Dies geschieht am eindrücklichsten in Kombination mit den anderen radiologischen Verfahren ( $\bullet$ Abb.1-3). Mithilfe solcher Bilder lernen Medizinstudenten auch, dass die besondere Aufgabe des Radiologen darin besteht, „den Vorhang aufzuziehen" und so - in Kenntnis der klinischen Daten des Patienten („dieser Apfel hatte einen Zusammenstoß bei hoher Geschwindigkeit“) das Unsichtbare sichtbar zu machen [8]. Die sich anschließenden Diskussionen mit tatsächlichen klinischen Fallbeispielen können die Rolle der Radiologie im interdisziplinären klinischen Entscheidungsfindungsprozess und somit auch ihren Beitrag für die Therapieoptimierung verdeutlichen. Diese humorvoll aufgelockerte Situation kann Bedingungen schaffen, in denen ein besonderes Interesse der Studenten besteht, mehr über die klinische Bedeutung der Radiologie zu verstehen. Dieser fruchtbare Boden sollte meines Erachtens genutzt werden, um den Studenten die modernen klinischen Aspekte der Radiologie nahe zu bringen. So können nachfolgende praktische Beispiele aus dem radiologischen Alltag sehr gut die Bedeutung einer modernen klinischen Radiologie unterstreichen (z. B. aus der Schlaganfallversorgung, der muskuloskelettalen und kardiovaskulären Bildgebung, bei der Polytraumaversorgung etc.) und überdies deutlich machen, dass die Radiologie sich zunehmend in klinische Entscheidungsprozesse vernetzt.

Frühzeitige praktische, aber unterhaltsame Erfahrungen mit der Radiologie machen angehende 

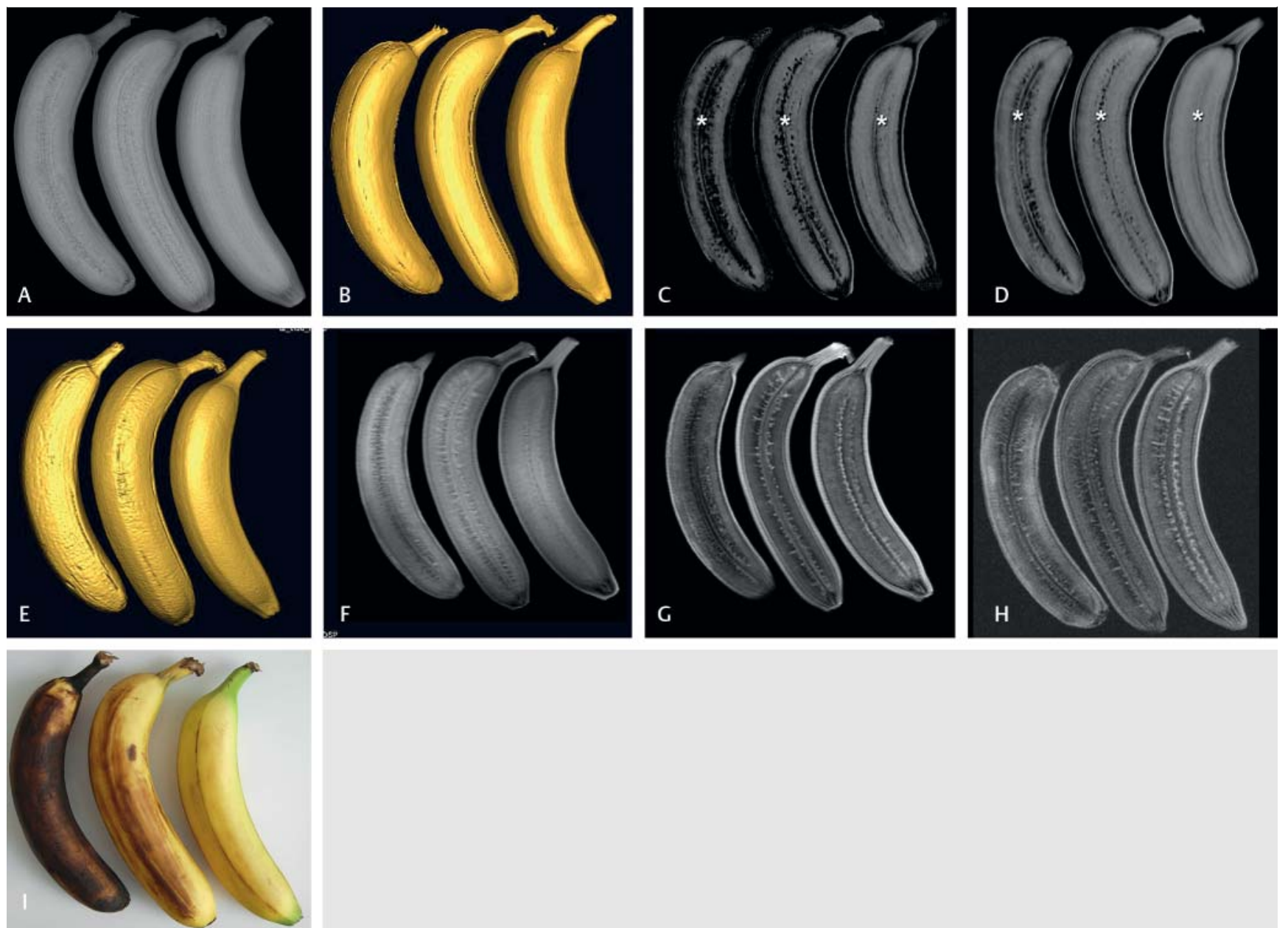

Abb. 1 Bildgebung mittels konventionellem Röntgen a, CT b-d sowie MRT e-h von 3 unterschiedlich reifen Bananen i. Die Röntgenaufnahme zeigt diskrete Konturveränderungen, wobei die überreife Banane (links in a) leicht unregelmäßig erscheint und einen hohen Luftgehalt aufweist. Dagegen enthält die Banane in der Mitte weniger Luft und die unreife Banane lediglich einige kleine Stellen (rechts in a). Die dreidimensionalen Rekonstruktionen b der CT-Aufnahmen (320 Detektorzeilen, Aquilion ONE, Toshiba) bestätigen die bereits im Röntgen nachgewiesenen Konturveränderungen a, bieten jedoch keinerlei Informationen zu Veränderungen im Luftgehalt. Koronare 5-mm-Minimum-Intensitäts-Projektionen (MinIP) c und 5-mm-Durchschnittsrekonstruktionen $\mathbf{d}$ zeigen den mit dem Reifegrad steigenden Luftgehalt überwiegend in der Mitte der Bananen (Sternchen). Die Luftmenge unmittelbar unter der Bananenschale erhöht sich mit der Reifung nicht. Eine mittlere Luftmenge, die nicht konfluiert (wie bei der

Ärzte mit unserem Fachgebiet vertraut und verbessern ihr radiologisches Grundwissen, wobei sie gleichzeitig auf diese Weise ein besseres Verständnis für die wichtige Aufgabe der Radiologie im Behandlungsprozess entwickeln [13]. Durch eine frühzeitige Auseinandersetzung mit dem Fach machen Studenten auch die Erfahrung, dass sie bereits während des Studiums Beiträge leisten können [14]. Der nach dem Einstieg mit den gezeigten Bildern vermittelte Lehrstoff umfasst auch physikalische Grundlagen und praktisches Wissen zu den verschiedenen bildgebenden Verfahren [15]. Dies führt zwanglos zu einer vertiefenden Diskussion der Vor- und Nachteile der Verwendung der verschiedenen bildgebenden Verfahren in der jeweiligen klinischen Situation, die in den nachfolgenden Lehrveranstaltungen im Detail besprochen werden. Damit werden aktive Lernprozesse angestoßen [16] und die Studenten überlegen selbst, welches diagnostische

Banane in der Mitte), scheint ein guter Indikator für den optimalen Reifungsgrad und damit die Essbarkeit zu sein. Der Luftgehalt ist am besten auf der MinIP-Aufnahme zu beurteilen c. Dreidimensionale Rekonstruktionen (Volumen-Rendering, e) der MRT (T2) bei 1,5T (Somatom Avanto, Siemens) bestätigen die Konturveränderungen, die bereits in der konventionellen Röntgenaufnahme a sowie im CT b zu sehen sind, und bieten darüber hinaus keine weiteren Informationen. Koronare T1-gewichtete (f Repetitionszeit [TR] $700 \mathrm{~ms}$, Echozeit [TE] 8,5 ms, 2 mm Schichtdicke) und T2-gewichtete (g TR 2935 ms, TE 120 ms, 2 mm Schichtdicke) Aufnahmen zeigen verminderte T1- und T2-Werte (f und $\mathbf{g}$ ) bei gleichzeitigem Wasserverlust durch die Reifung (h TIRM TR 9670 ms, TE 114 ms, 2 mm Schichtdicke). Schäden, wie sie beispielsweise beim Transport der Früchte entstehen, werden als Hauptursache für die T2-Verminderung während der Reifung angesehen [20].

Verfahren am besten geeignet ist, um eine bestimmte klinische Fragestellung zu beantworten. Dies entspricht auch dem Ziel der Röntgenverordnung, durch die Prüfung der rechtfertigenden Indikation zu entscheiden, ob Röntgenverfahren zur Beantwortung einer medizinischen Fragestellung durch andere bildgebende Methoden ersetzt werden können [12].

Anschließend erfolgt das Abwägen möglicher therapeutischer Maßnahmen. Durch einen derartigen Lehransatz, der eigene Entscheidungsprozesse bei den Studenten in Gang setzt, sind wir auch eher gegen die Versuchung gefeit, mit Faktenwissen überfrachtete Lehrpläne aufzustellen, dabei aber die "großen Ideen“ nicht zu vermitteln. Dies haben Gunderman et al. zu Recht als eine Gefahr identifiziert, die der Vermittlung von echter Fachkompetenz entgegensteht [17]. Angesichts der kurzen Halbwertzeit medizinischen Wissens [18] müssen sich angehende Ärzte 

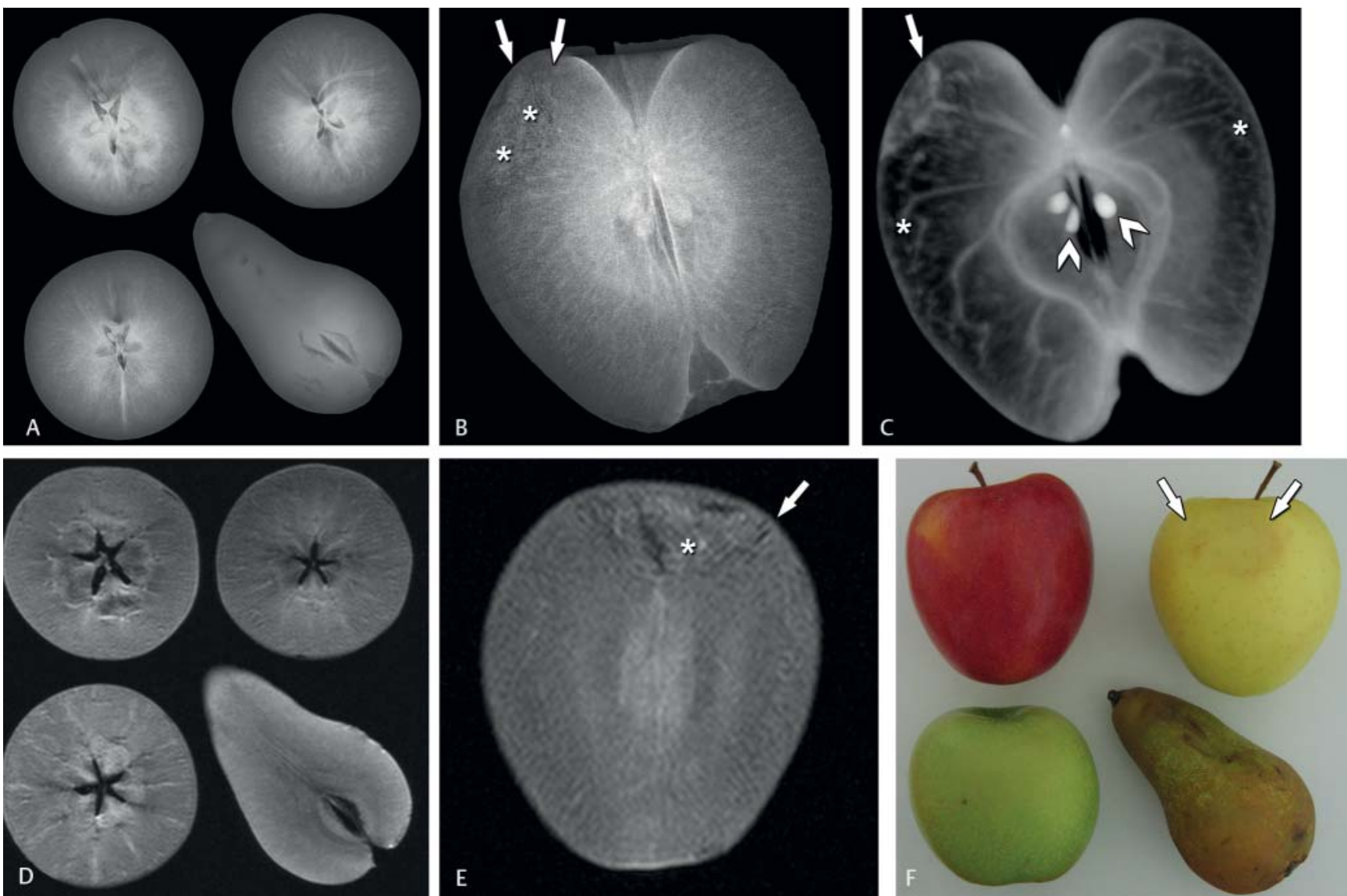

Abb. 2 Konventionelle Röntgenaufnahmen (a und b), CT-Aufnahme c sowie MRT-Bilder ( $\mathbf{d}$ und e) von 2 intakten Äpfeln und einem Apfel mit einer Druckstelle (f Pfeile) nach einem Fall aus großer Höhe (vom Küchentisch) sowie einer Birne (a, d und f). Die kraniokaudale Röntgenübersicht zeigt die 3 Äpfel und die Birne, nicht jedoch die Druckstelle am Apfel a. Die laterale Röntgenaufnahme des Apfels mit der Druckstelle (Pfeile in $\mathbf{f}$ ) zeigen die Druckstelle in Form durchscheinender Linien (Pfeil in $\mathbf{b}$ ) sowie Zerstörung der inneren Organisationsstruktur des Apfels (Sternchen in b). Eine koronare 5mm-Minimum-Intensitätsprojektion der CT-Aufnahme zeigt sehr schön Details der hyperdensen Druckstelle (Pfeil in c), die dem Erscheinungsbild einer Kompressionsfraktur des Wirbelkörpers sehr ähnlich ist. Im Vergleich zum

konventionellen Röntgen zeigt die CT-Aufnahme deutlicher die symmetrische Binnenstruktur des Apfels (Sternchen in c) sowie die Bestandteile des Kerngehäuses wie die Kerne (Pfeilspitzen in c). Die axiale T2-gewichtete Sequenz (d TR 6500 ms, TE 100 ms, 4 mm Schichtdicke) gibt einen Überblick über die Unterschiede zwischen den 3 Apfelsorten d. Diese T2-gewichtete Aufnahme zeigt auch sehr schön die sehr viel größere Homogenität der Birne (rechte untere Ecke in d) im Vergleich zu den 3 Äpfeln. Eine koronare T2-gewichtete Aufnahme (e TR $6500 \mathrm{~ms}$, TE $100 \mathrm{~ms}, 2 \mathrm{~mm}$ Schichtdicke) zeigt die Druckstelle des Apfels als hypointense Linie (Pfeil in e) sowie helle Pünktchen, die Flüssigkeitsansammlungen entsprechen könnten (Sternchen in e).

stattdessen Strategien aneignen, mit denen sie insbesondere auch neues Wissen erfassen und bewerten können, gemäß dem Motto „Die Guten ins Töpfchen, die Schlechten ins Kröpfchen“. Die hier vorgeschlagene Strategie des pointiert eingesetzten Humors im radiologischen Unterricht muss nicht im Widerspruch zum in Entwicklung befindlichen „Nationalen kompetenzbasierten Lernzielkatalog Medizin“ stehen, da die nach der humoristischen Einleitung vorgestellten praktische Beispiele aus dem radiologischen Alltag die formellen Kriterien eines Lernzielkatalogs mit spezifischen und übergeordneten Lernzielen gut erfüllen können. Durch die zunehmende Modularisierung der Lehre im Medizinstudium und eine organbezogene Strukturierung gehen die Identität und Wahrnehmbarkeit des Faches Radiologie durch die Studenten nachhaltig zurück. Diese Entwicklung ist eine der wichtigsten Herausforderungen für unser Fachgebiet. Auch deshalb muss es uns gelingen, die Sichtbarkeit und Nachhaltigkeit der integrativ angebotenen radiologischen Veranstaltungen durch eine ungewöhnlich, z. B. humoristische, Herangehensweise zu verbessern.

Nyce et al. haben es wie folgt auf den Punkt gebracht: „Das Ziel der Radiologie und damit der radiologischen Ausbildung besteht vor allem darin, den Patienten zu helfen. Hierzu müssen die Lehrenden selbst die bestmöglichen Beurteilungsparadigmen entwickeln und den Studierenden vermitteln. Nur so kann die Lehre im Fachgebiet Radiologie das Verständnis der Studenten bestmöglich bereichern." [8] Bilder, mit denen wir unsere Studenten erst zum Lachen und dann zum Nachdenken bringen, sind vielleicht genau das richtige Mittel zur Vermittlung dieses Anliegens. Nach meiner persönlichen Erfahrung findet dieses Vorgehen in ähnlicher Art wie digitale Fallsammlungen [19] bei den Studierenden Anklang und ich kann es deshalb sehr weiterempfehlen. Dies verstehe ich auch als Anregung, eigene Bilder von ungewöhnlichen Objekten einzubringen - damit können wir nicht nur die Lehre verbessern, sondern auch die Lernatmosphäre im dunklen Hörsaal aufhellen.

\section{Widmung}

$\nabla$

Dieses Manuskript ist Professor Bernd Hamm zum 60. Geburtstag gewidmet. 

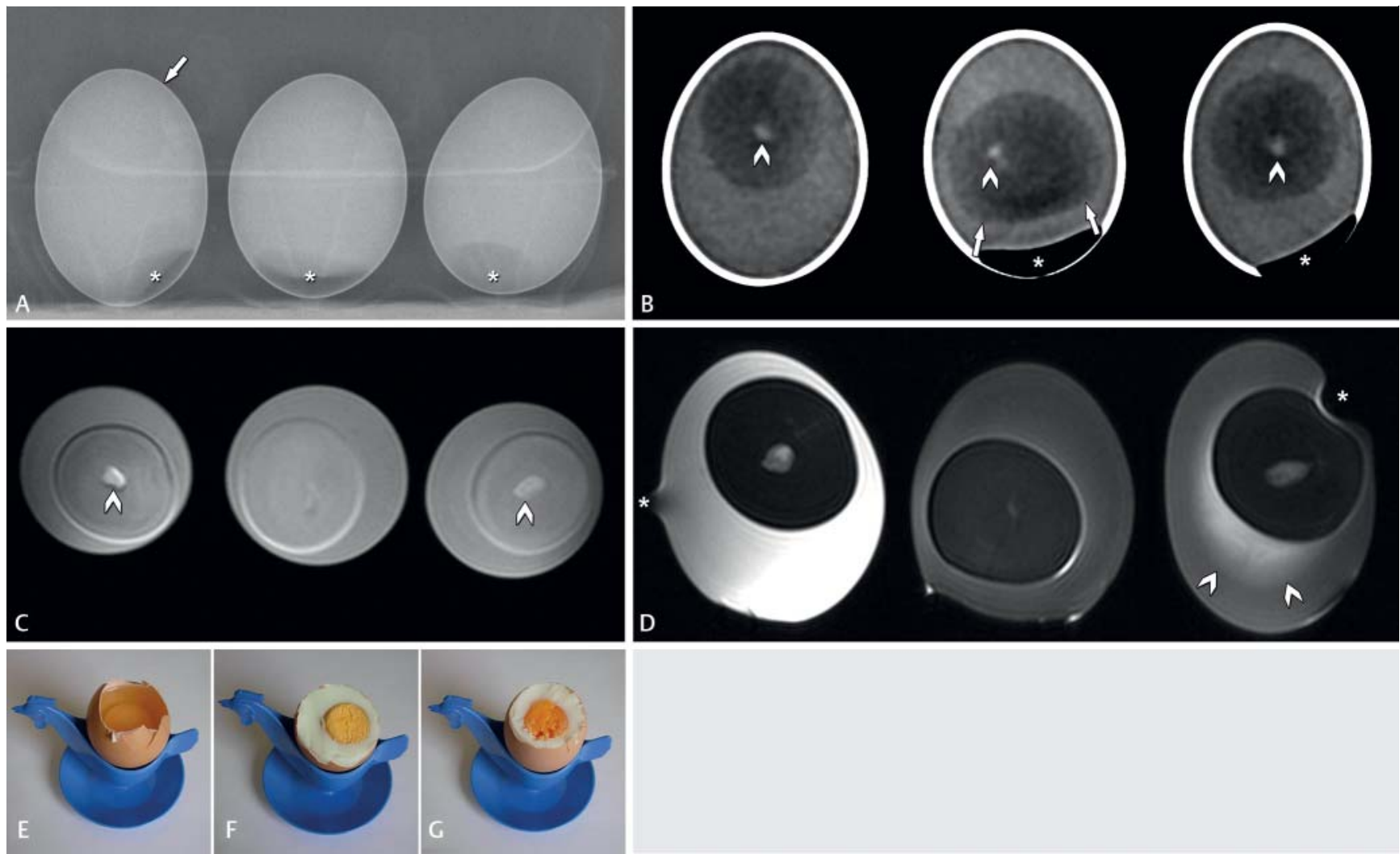

Abb. 3 Röntgenaufnahme $\mathbf{a}$, CT-Bild $\mathbf{b}$ und MRT-Aufnahme ( $\mathbf{c}$ und $\mathbf{d}$ ) von einem rohen e sowie einem hart gekochten $\mathbf{f}$ und einem weich gekochten $\mathrm{Ei}$ g. Eine laterale Röntgenaufnahme zeigt die Eier (im Eierkarton) mit guter Darstellung der Schale (Pfeil) sowie der Luftkammer (Sternchen in a); Aussagen über den Kochzustand können anhand der Aufnahme nicht getroffen werden. Die Schale sowie die Luftkammer (Sternchen in b) lassen sich selbstverständlich auch auf der koronaren CT-Rekonstruktion abgrenzen. Ebenfalls ersichtlich ist eine Abflachung des Eidotters, was auf ein hart gekochtes Ei hindeuten könnte (Pfeile in b). Die beiden anderen Eier unterscheiden sich jedoch kaum und weisen vergleichbare Dichten des Eigelbs und des Eiweißes auf. Auch die Keimscheibe (Pfeilspitzen in b) erlaubt im CT keine Unterscheidung zwischen dem rohen (links), dem hart gekochten (Mitte) und dem weich gekochten Ei (rechts). Dagegen kann im axialen T1-

\section{Literatur}

1 Berk RA. Humor as an instructional defibrillator. The Journal of health administration education 2007; 24: 97-116

2 Berk RA. Plenary Address: Humour in Medicial Education is like a Box of Chocolates. Berlin: Association for Medical Eduction in Europe (AMEE); 2001

3 Pilcher H. A better class of heckle. Nature 2010; 467: 530

4 Schultz WW, van Andel P, Sabelis I et al. Magnetic resonance imaging of male and female genitals during coitus and female sexual arousal. BMJ 1999; 319: $1596-1600$

5 Witcombe B, Meyer D. Sword swallowing and its side effects. BMJ 2006; 333: $1285-1287$

6 Squire LF, Blotnick V, Becker JA. Self-instruction in radiology for medical students. Radiology 1972; 105: 681-684

7 Berk RA. Plenary Address: Humour in Medicial Education is like a Box of Chocolates. Berlin: Association for Medical Eduction in Europe (AMEE); 2001

8 Nyce JM, Steele JS, Gunderman RB. Bridging the knowledge divide in radiology education. Radiology 2006; 239: 629-631

9 Gunderman RB, Hill DV. Student Concerns and Misconceptions about A Career in Radiology. Acad Radiol 2012; 19: 366-368

10 Zuppinger A. Ausbildung in Radiologie. Fortschr Röntgenstr 1977; 127: 585-591

11 Collins J. Keys to educator effectiveness in radiology. Acad Radiol 2006; 13: $641-643$

gewichteten MRT-Bild (c TR 700 ms, TE 8,5 ms, 3 mm Schichtdicke) die hyperintense Keimscheibe des rohen und des weich gekochten Eis (Pfeilspitzen in c) von der Keimscheibe des hart gekochten Eis unterschieden werden, die sich isointens zum Eigelb darstellt (mittleres Ei in c). Ähnliches Signalverhalten zeigt die Keimscheibe in der koronaren T2-gewichteten Aufnahme (d TR 4500 ms, TE 100 ms, 3 mm Schichtdicke). Die T2-gewichtete Aufnahme zeigt auch Unterschiede im Eiweiß, das im Rohzustand die höchste Signalintensität aufweist (linkes Ei in d) und die geringste Intensität im hart gekochten Ei (mittleres Ei in d). Ein köstliches Merkmal von weich gekochten Eiern - das noch leicht flüssige Eiweiß um den Dotter - ist in der T2Wichtung ebenfalls erkennbar (Pfeilspitzen in d). Weiterhin treten im MRT am Übergang des Eis zur umgebenden Luft Artefakte auf (Sternchen in d).

12 Heindel W, Adam G. Magnetresonanz-Verfahren: Qualitätssicherung in Krankenversorgung, Lehre und Forschung. Fortschr Röntgenstr 2003; 175: $58-60$

13 Branstetter BFt, Faix LE, Humphrey AL et al. Preclinical medical student training in radiology: the effect of early exposure. Am J Roentgenol 2007; 188: W9-W14

14 Channin DS. Early contributions of medical students, interns, and residents to the development of radiology. Am J Roentgenol 1993; 160: 647-648

15 Gunderman RB, Stephens $C D$. Teaching medical students about imaging techniques. Am J Roentgenol 2009; 192: 859-861

16 Zou L, King A, Soman S et al. Medical students' preferences in radiology education a comparison between the Socratic and didactic methods utilizing powerpoint features in radiology education. Acad Radiol 2011; 18: 253-256

17 Gunderman R, Williamson K, Fraley R et al. Expertise: implications for radiological education. Acad Radiol 2001; 8: 1252 - 1256

18 Kressel HY. Scientific dialogue: vestige of the past or hope for the future? Radiology 2011; 258: 12 -14

19 Heye T, Kurz P, Eiers $M$ et al. Eine radiologische Fallsammlung mit interaktivem Charakter als neues Element in der studentischen Ausbildung. Fortschr Röntgenstr 2008; 180: 337-344

20 Ribeiro FZ, Marconcini LV, de Toledo IB et al. Nuclear magnetic resonance water relaxation time changes in bananas during ripening: a new mechanism. J Sci Food Agric 2010; 90: 2052-2057 